\title{
Src regulates phorbol 12-myristate 13-acetate-activated PKC-induced migration via Cas/Crk/Rac1 signaling pathway in glioblastoma cells
}

\author{
NAOKO NOMURA ${ }^{1,2}$, MOTOHIRO NOMURA ${ }^{3}$, KAZUHISA SUGIYAMA ${ }^{1}$ and JUN-ICHIRO HAMADA ${ }^{4}$ \\ ${ }^{1}$ Department of Ophthalmology and Visual Science, Kanazawa University Graduate School of Medical Science, Kanazawa; \\ ${ }^{2}$ Department of Ophthalmology and Visual Science, Yokohama City University School of Medicine, Yokohama; \\ ${ }^{3}$ Department of Neurosurgery, Yokohama Sakae Kyosai Hospital, Yokohama; ${ }^{4}$ Department of Neurosurgery, \\ Kanazawa University Graduate School of Medical Science, Kanazawa, Japan
}

Received April 20, 2007; Accepted June 1, 2007

\begin{abstract}
In this study, we demonstrate that phorbol 12myristate 13-acetate (PMA)-activated protein kinase C (PKC) induced migration in A172 glioblastoma cells via Src. PMA treatment induced tyrosine phosphorylation of Crk-associated substrate (Cas) and formation of a complex with Crk, followed by Rac1 activation, a downstream effector of Cas/Crk complex. These effects were blocked by a tyrosine kinase inhibitor (PP2) or Src small interfering RNA (siRNA), indicating that Src was involved in the PMA-induced activation of Cas/ Crk/Rac1 signaling pathway. An immunohistochemical study showed that after PMA treatment, Cas, Crk and Rac1 translocated into lamellipodia. Tyrosine phosphorylated Cas was also detected at the periphery of the cells, where focal complexes were prominent. These results indicated that signaling of Cas, Crk and Rac1 might be involved in PMAinduced cytoskeletal reorganization. Translocation of Rac1 to the cell membrane is known to be dependent on phosphorylation of tyrosine-221 residue of Crk. We demonstrated that PMA induced phosphorylation of Crk, and this phosphorylation was blocked by PP2 or Src siRNA. These results indicated that Src might regulate the subcellular localization of Rac1 through phosphorylation of Crk. We propose that PMA-induced migration was dependent on activation of $\mathrm{PKC} / \mathrm{Src} / \mathrm{Cas} /$ Crk/Rac1 signaling pathway via modulating cytoskeletal reorganization during glioblastoma cell migration.
\end{abstract}

Correspondence to: Dr Motohiro Nomura, Department of Neurosurgery, Yokohama Sakae Kyosai Hospital, 132 Katsura-cho, Sakae-ku, Yokohama, Kanagawa 247-8581, Japan

E-mail: nomura413jp@yahoo.co.jp

Abbreviations: PMA, phorbol 12-myristate 13-acetate; PKC, protein kinase C; siRNA, small interfering RNA; BIS, bisindolylmaleimide I; PP2, 4-Amino-5-(4-chlorophenyl)-7-(t-butyl)pyrazolo[3,4-d] pyrimidine

Key words: glioblastoma, migration, protein kinase C, PMA, Src

\section{Introduction}

Glioblastoma has high cell proliferative ability and a propensity to infiltrate into surrounding brain. These characteristics prohibit complete surgical resection of the tumor (1). A better understanding of the processes controlling glioblastoma migration is therefore required to improve the efficacy of treatment of this disease.

Several factors and kinases have been reported to be involved in promoting glioblastoma cell migration (2). Among them, overexpressed or hyperactive protein kinase $\mathrm{C}$ (PKC) is the most distinctive characteristics of this malignant brain tumor $(3,4)$. PKC is a serine/threonine kinase that stimulates several protein tyrosine kinases. It is responsible for mediating several signal transduction pathways which result in cell migration, adhesion, and proliferation (2). In this study, we showed the involvement of Src in phorbol 12-myristate 13-acetate (PMA)-activated PKC-induced lamellipodia formation and subsequent migration of glioblastoma cells.

Src, a non-receptor tyrosine kinase with a molecular weight of $60 \mathrm{kDa}$, is involved in cell proliferation, adhesion, spreading and motility through tyrosine phosphorylation of focal adhesion substrates such as focal adhesion kinase (FAK) and Crk-associated substrate (Cas) $(5,6)$.

Cas is known to be an adapter protein, and was isolated from cells transformed with the v-Crk or v-Src gene (7). Cas contains an N-terminal Src homology 3 (SH3) domain that binds to FAK and a C-terminal Src-binding domain (SBD) that includes a binding site for the SH3 domain of Src family kinases. Lying between the SH3 domain and the SBD is a substrate domain characterized by 15 Tyr-X-X-Pro (YxxP) motifs. The phosphorylated YxxP motifs serve as docking sites for SH2-mediated binding to adapter proteins of the Crk family (8). Cas/Crk coupling is implicated as a critical step in promoting relevant downstream signaling events, including activation of the small GTPase Rac1 $(8,9)$. Rac, a member of the Rho family of small GTPases, controls the signal transduction pathway linking membrane receptors to the assembly and disassembly of the actin cytoskeleton and associated integrin adhesion complexes (9-11).

Although there are several studies showing that PKC induces cell migration, the mechanism of PKC-induced cell 
migration has not been completely clarified. In this study, we investigated the mechanism of PMA-activated PKC-induced migration of glioblastoma cells focusing on the Src pathway. We demonstrated that PMA-induced migration of glioblastoma cells was dependent on Src. The PMA-induced migration of glioblastoma cells was shown to be a consequence of Srcdependent Cas/Crk complex formation, Rac1 activation, and translocation of Rac1 to the cell periphery followed by formation of lamellipodia and focal complexes.

\section{Materials and methods}

Reagents and antibodies. PMA and bisindolylmaleimide I (BIS) were purchased from Sigma-Aldrich Corporation (St. Louis, MO). 4-Amino-5-(4-chlorophenyl)-7-(t-butyl) pyrazolo[3,4-d]pyrimidine (PP2) was purchased from Calbiochem (San Diego, CA). The primary antibodies used were rabbit anti-phospho-Crk (Tyr-221) polyclonal antibody (Cell Signaling Technology, Beverly, MA); mouse anti-Cas monoclonal antibody (BD Biosciences, Franklin Lakes, NJ); mouse anti-Crk monoclonal antibody (BD Biosciences); rabbit anti-phospho-Cas (Tyr-165) polyclonal antibody (Cell Signaling Technology); mouse anti-Src monoclonal antibody (Santa Cruz, Santa Cruz, CA); mouse anti-phosphotyrosine (PY20) monoclonal antibody (Upstate Biotechnology, Temecula, CA); mouse anti-Rac1 antibody (Upstate Biotechnology); mouse anti-vinculin monoclonal antibody (Sigma-Aldrich Coporation); and rabbit anti-phospho-Src (Tyr-418) polyclonal antibody (Biosource International, Camarillo, CA).

Cell lines. A172 cells were obtained from American Type Culture Collection (Manassas, VA). The cells were maintained in Dulbecco's modified Eagle's medium (DMEM) (Bio Whittaker, Rockland, ME) supplemented with $10 \%$ fetal bovine serum (FBS; Atlanta Biologicals, Norcross, GA), $100 \mathrm{U} / \mathrm{ml}$ penicillin, $100 \mu \mathrm{g} / \mathrm{ml}$ streptomycin, and $2 \mathrm{mM}$ glutamine (Gibco BRL, Grand Island, NY) in a humidified atmosphere containing $5 \% \mathrm{CO}_{2}$ and $95 \%$ air at $37^{\circ} \mathrm{C}$.

Activation of PKC and inhibition of kinase activity. To activate PKC, 100 nM PMA was used for all experiments. PMA was added to the culture medium and the cells were incubated for various time intervals. To analyze the roles of various protein kinase activities, specific inhibitors were used, and the migration of cells and expression of proteins were analyzed. BIS and PP2 were used for inhibition of PKC and Src activity, respectively. BIS or PP2 at the concentration of 5 or $10 \mu \mathrm{M}$, respectively, was added to the culture medium $30 \mathrm{~min}$ prior to the addition of PMA.

Migration assay. The cells were seeded in 12-well culture dishes (BD Sciences) and cultured until they became subconfluent. The cells were then incubated with serum-free culture medium for $24 \mathrm{~h}$, and were scraped with a $200-\mu 1$ micro-pipet tip and washed with PBS. The cells were incubated with serum-free culture medium for an additional $16 \mathrm{~h}$ with 100 nM PMA or vehicle. When specific inhibitors were used, an appropriate concentration of each inhibitor was added to the culture medium 30 min before PMA treatment. After $16 \mathrm{~h}$ of incubation, the cells were photographed, and the migrated area was measured using NIH image software.

Transfection of siRNA into glioblastoma cells. Small interfering RNA (siRNA) for Src was purchased from Upstate Biotechnology. For control experiments, control siRNA-A (Santa Cruz) was used. Reagents for transfection (Lipofectamine 2000 and Opti-MEM I reduced serum medium) were obtained from Invitrogen (Invitrogen, Carlsbad, CA). Transfection of siRNA into the glioblastoma cells was done according to the manufacturer's instructions. After $48 \mathrm{~h}$ of incubation of the cells with $100 \mathrm{nM}$ of siRNA, the cells were cultured for an additional $24 \mathrm{~h}$ with serum-free medium, and then subjected to further experiments.

Western blot analysis. Total cells were harvested from each culture condition at the appropriate time, and protein was extracted using a lysis buffer containing $1 \%$ Triton X-100, $150 \mathrm{mM} \mathrm{NaCl}, 5 \mathrm{mM}$ EDTA, $50 \mathrm{mM}$ sodium fluoride, $1 \mathrm{mM}$ sodium orthovanadate, $5 \mu \mathrm{g} / \mathrm{ml}$ phenylmethylsulfonyl fluoride, $2 \mu \mathrm{g} / \mathrm{ml}$ aprotinin, $5 \mu \mathrm{g} / \mathrm{ml}$ leupeptin, and $2 \mu \mathrm{g} / \mathrm{ml}$ pepstatin. The samples were centrifuged at $14000 \mathrm{rpm}$ for $30 \mathrm{~min}$ at $4^{\circ} \mathrm{C}$. The extracts were stored at $-80^{\circ} \mathrm{C}$ until use. Protein concentration was determined using the BCA assay (Pierce, Rockford, IL).

For Western blot analysis, equal amounts of protein were electrophoresed on SDS-PAGE gels, transferred to nitrocellulose membranes (Trans-Blot transfer medium $0.45 \mu \mathrm{m}$, Bio-Rad, Hercules, CA) and stained with Ponceau S (SigmaAldrich Corporation). After confirmation of protein transfer, proteins were detected with specific antibodies. All primary antibodies were used at 1:1000 dilution. Actin protein was detected as a control with mouse anti-human B-actin monoclonal antibody used at 1:10000 (Chemicon, Temecula, CA). Sheep anti-mouse IgG or donkey anti-rabbit IgG horseradish peroxidase-linked secondary antibodies (Amersham, Piscateway, NJ) at 1:4000 dilution were used. Protein detection was performed using SuperSignal West Femto maximum sensitivity substrate (Pierce) and signals were visualized using Hyperfilm ECL (Amersham).

Immunoprecipitation. Immunoprecipitation was performed as described previously (12). Precleared protein G-Sepharose (Pharmacia Biotech, Uppsala, Sweden) was preincubated with antibodies for immunoprecipitation. Then cell lysates $(750 \mu \mathrm{g})$ were incubated with protein G-Sepharose at $4^{\circ} \mathrm{C}$ with constant rotation. The beads were washed seven times with lysis buffer, and then boiled at $95^{\circ} \mathrm{C}$ for $5 \mathrm{~min}$. The sample buffer containing immunoprecipitated protein was electrophoresed on an SDS-PAGE gel and the protein was transferred to a nitrocellulose membrane by electroblotting. After confirmation of protein transfer, specific proteins were detected with specific antibodies used at 1:1000 dilution.

Fluorescence immunohistochemistry. Cells were plated in 8-well culture dishes (BD Sciences) and incubated overnight. After attachment, the cells were incubated with serum-free culture medium for $24 \mathrm{~h}$. Then $100 \mathrm{nM}$ PMA was added to the serum-free culture medium and the cells were incubated for $60 \mathrm{~min}$. When specific inhibitors were used, an appropriate 
A

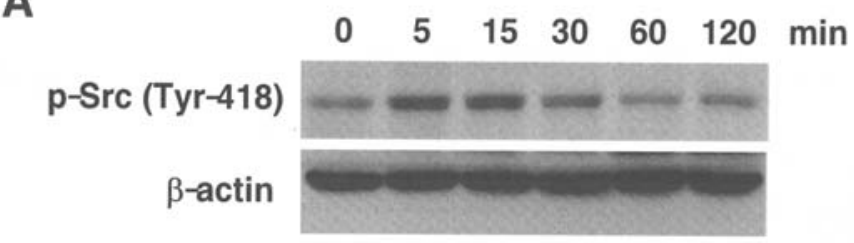

B

\section{p-Src (Tyr-418) \\ $\beta$-actin}

Figure 1. Effect of PMA on expression and activation of Src in glioblastoma cells. (A) A172 glioblastoma cells were treated with PMA for the indicated times. (B) For inhibition experiments, BIS or PP2 was added to serumstarved cells $30 \mathrm{~min}$ prior to PMA stimulation. Then the cells were stimulated with PMA or vehicle for $15 \mathrm{~min}$. Expression and activation of Src were examined by Western blot analysis. Src activation was evaluated with an anti-phospho-Src (Tyr-418) antibody. The amount of total Src or $B$-actin was determined by reprobing the membrane after stripping.

concentration of each inhibitor was added to the culture medium 30 min before PMA stimulation. After incubation, the cells were fixed with $3.7 \%$ paraformaldehyde, and permeabilized with $0.2 \%$ Triton $\mathrm{X}-100$. After blocking with $2 \%$ BSA, the cells were incubated with specific primary antibodies at a dilution of 1:100. Then the cells were incubated with fluorescence-conjugated secondary antibodies at 1:200 dilution, and mounted. The primary antibody labeled with Alexa fluor 488 phalloidin (Invitrogen) was used for detection of F-actin (labeled green) under a fluorescence microscope. The secondary antibodies used were 594 Alexa fluor-labeled goat anti-mouse antibody (Invitrogen) or 488 Alexa fluorlabeled goat anti-rabbit antibody (Invitrogen). The secondary antibodies were labeled red or green under a fluorescence microscope, respectively.

Assay of Racl activation. The Rac-GTP pull down assay was performed by using a Rac1 activation assay kit (Upstate Biotechnology) according to the manufacturer's instructions. In brief, the cell lysates were incubated with PAK-1 PBD agarose to bind Rac1-GTP. The proteins bound to PAK-1 PBD were separated by SDS-PAGE, transferred to a nitrocellulose membrane and probed with Rac1 antibody.

Statistical analysis. All experiments were performed at least 3 times. The data were expressed as the mean \pm standard error of the mean (SEM). Probability (P) was calculated using a Student's t-test. P-values $<0.05$ were considered significant.

\section{Results}

PMA-induced migration of glioblastoma cells was blocked by Src inhibition. To evaluate the relationship between PMAinduced migration of glioblastoma cells and Src activity, Src activation was assessed by using a specific antibody for Tyr418 of Src, which can detect an activated form of Src $(13,14)$.
A
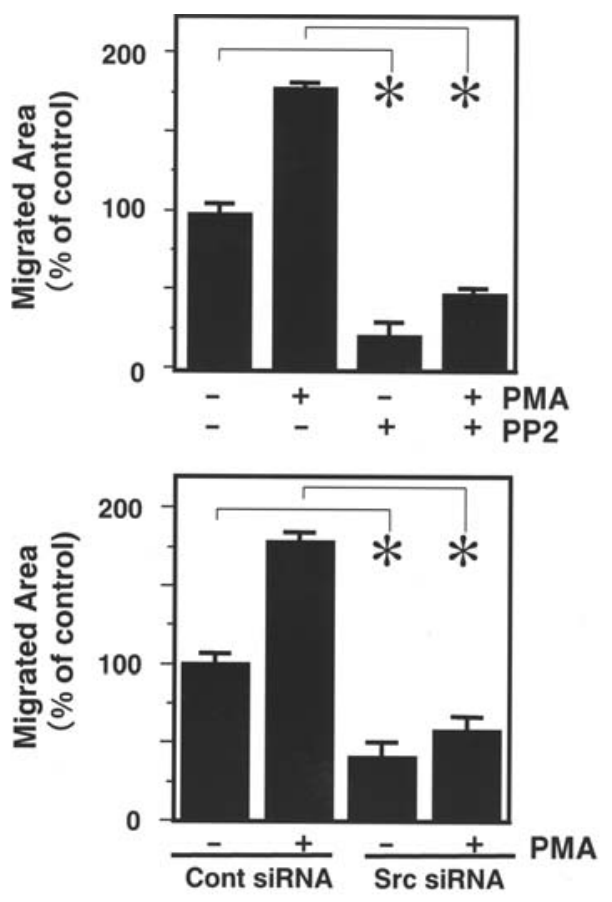

B

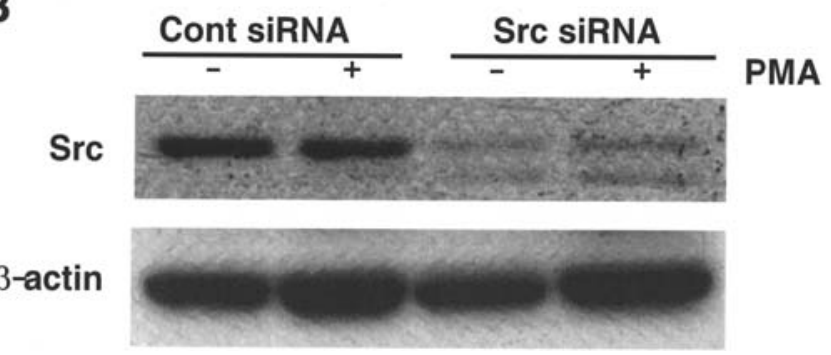

Figure 2. Effect of Src on PMA-induced migration. (A) Cells pretreated with inhibitors or vehicle were incubated without serum for $24 \mathrm{~h}$ and wounded with a micro-pipet tip. PP2 was added 30 min prior to PMA treatment (upper panel). Cells transfected with control or Src siRNA were incubated without serum for $24 \mathrm{~h}$ and wounded (lower panel). Then the cells were stimulated with PMA. After 16 h, migration was evaluated. Results from three independent experiments are presented. Migration was expressed as the percentage of unstimulated cells at $16 \mathrm{~h} .{ }^{*} \mathrm{p}<0.05$, compared to the control. (B) Cells transfected with control or Src siRNA were stimulated with PMA or vehicle for $15 \mathrm{~min}$. Expression of Src was examined by Western blot analysis.

As indicated in Fig. 1A, PMA treatment rapidly induced activation of Src within 5 min in A172 glioblastoma cells. The elevation of activated Src persisted until $30 \mathrm{~min}$. This activation was inhibited by pretreatment of the cells with a Src tyrosine kinase inhibitor (PP2) or PKC-inhibitor (BIS) (Fig. 1B, lanes 3 and 4). These results indicated that PMAinduced Src activation was dependent on PKC activation.

Next to analyze the role of Src activation in PMA-induced migration of glioblastoma cells, we assessed cell migration using a wound healing assay with PP2 treatment of PMAtreated glioblastoma cells. As shown in Fig. 2A, PP2 inhibited the PMA-induced migration of glioblastoma cells to $26 \%$ compared to the control level (upper panel). However, the possible inhibitory effects of PP2 on tyrosine kinases other than Src tyrosine kinase cannot be excluded. To achieve more specific inhibition, we analyzed the migration of Src siRNAtransfected cells. Transfection of Src siRNA was confirmed to decrease the basal expression of Src protein to $12 \%$ of the 
A

p-Cas (Tyr-165)

Cas
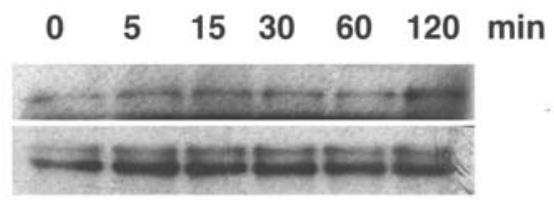

B

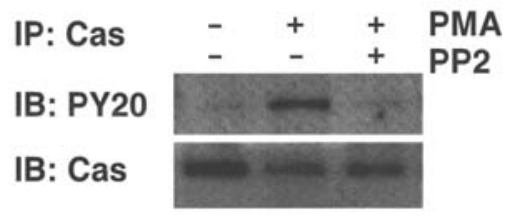

C

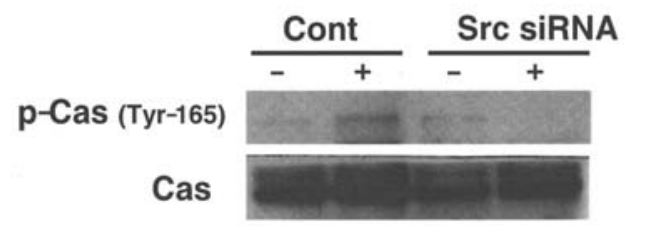

D

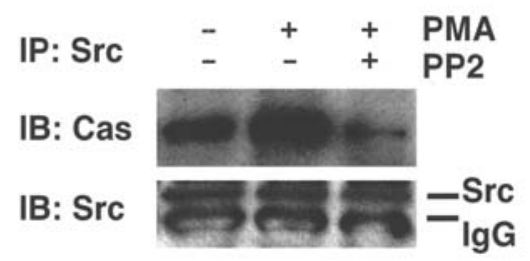

Figure 3. Effect of PMA on tyrosine phosphorylation of Cas in glioblastoma cells. (A) The cells were treated with PMA for the indicated times. Expression and tyrosine phosphorylation of Cas were examined by Western blot analysis. Tyrosine phosphorylation of Cas was evaluated with an antiphospho-Cas (Tyr-165) antibody (upper). The amount of total Cas was determined by reprobing the membrane after stripping (lower). (B) For immunoprecipitation experiments, PP2 was added to serum-starved cells 30 min prior to PMA stimulation. Then the cells were stimulated with PMA or vehicle for $60 \mathrm{~min}$. The protein extracted was immunoprecipitated for Cas and then immunoblotted for phosphotyrosine (PY20) (upper) or Cas (lower). (C) The cells transfected with control or Src siRNA were stimulated with PMA or vehicle for $60 \mathrm{~min}$. Expression and tyrosine phosphorylation of Cas were examined by Western blot analysis. (D) PP2 was added to serum-starved cells 30 min prior to PMA stimulation. Then the cells were stimulated with PMA or vehicle for 60 min. The extracted protein was immunoprecipitated for Src and then immunoblotted for Cas (upper) or Src (lower).

control by Western blot analysis (Fig. 2B). Src siRNA also inhibited the PMA-induced migration of glioblastoma cells to $32 \%$ of the control level (Fig. 2A, lower panel). These results indicated that $\mathrm{Src}$ was required for PMA-induced migration of glioblastoma cells.

PMA-induced tyrosine phosphorylation of Cas was mediated by $\operatorname{Src}$. It has been reported that integrin-mediated tyrosine phosphorylation of Cas is largely dependent on the presence of c-Src (15). To test whether Cas is tyrosine phosophorylated by PMA via Src activation, we examined the tyrosine phosphorylation of Cas by Western blot analysis using anti-phospho Cas (Tyr-165) antibody which specifically recognizes phosphorylated YxxP tyrosines in the Cas substrate domain. As shown in Fig. 3A, Cas began to be tyrosine phosophorylated 5 min after PMA stimulation and the phosphorylation persisted for at least $120 \mathrm{~min}$. The phosphorylation of Cas was abolished

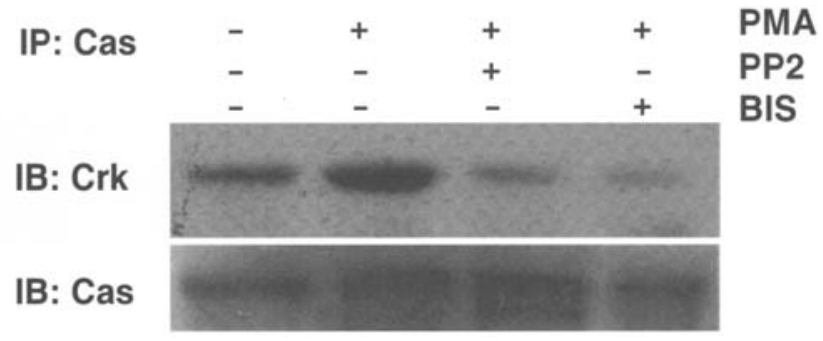

Figure 4. Effect of PMA on association of Cas with Crk in glioblastoma cells. For immunoprecipitation experiments, BIS or PP2 was added to serum-starved cells 30 min prior to PMA stimulation. Then the cells were stimulated with PMA or vehicle for $60 \mathrm{~min}$. The extracted protein was immunoprecipitated for Cas and then immunoblotted for Crk.

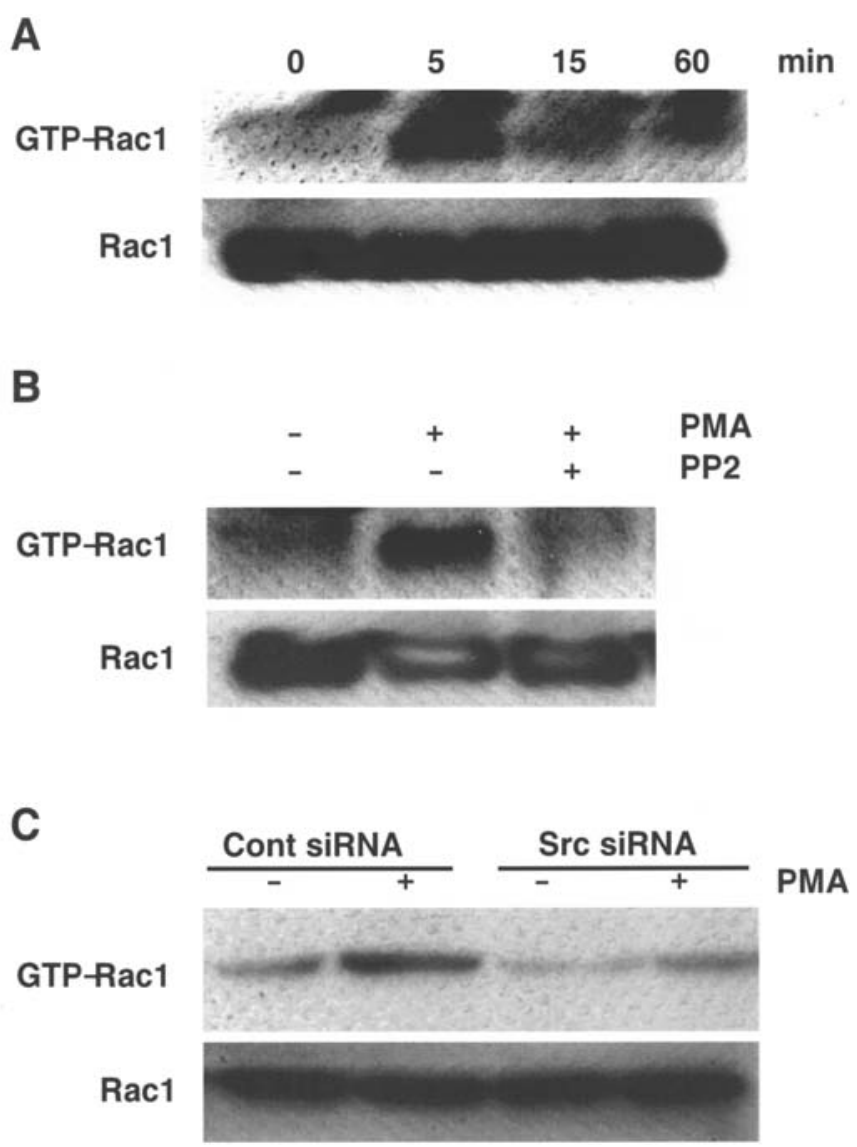

Figure 5. Effect of PMA on activation of Rac1 in glioblastoma cells. (A) Cells were treated with PMA for the indicated times. (B) For inhibition experiments, PP2 was added to serum-starved cells 30 min prior to PMA stimulation. Then the cells were stimulated with PMA or vehicle for $5 \mathrm{~min}$. Activated Rac1 was isolated in pull-down assays as described in Materials and methods. Bound GTP-Rac1 was detected by Western blot analysis and indicated as GTP-Rac1 (upper). Whole cell lysates were also probed for total Rac1 (lower). (C) Cells transfected with control or Src siRNA were incubated without serum for $24 \mathrm{~h}$. Then the cells were stimulated with PMA or vehicle for $5 \mathrm{~min}$.

by BIS (data not shown), indicating that PMA-induced tyrosine phosphorylation of Cas was a consequence of PKC activation.

Then, to observe the tyrosine phosphorylation of Cas in more detail, immunoprecipitation was done as described in Materials and methods. As shown in Fig. 3B, Cas protein was 


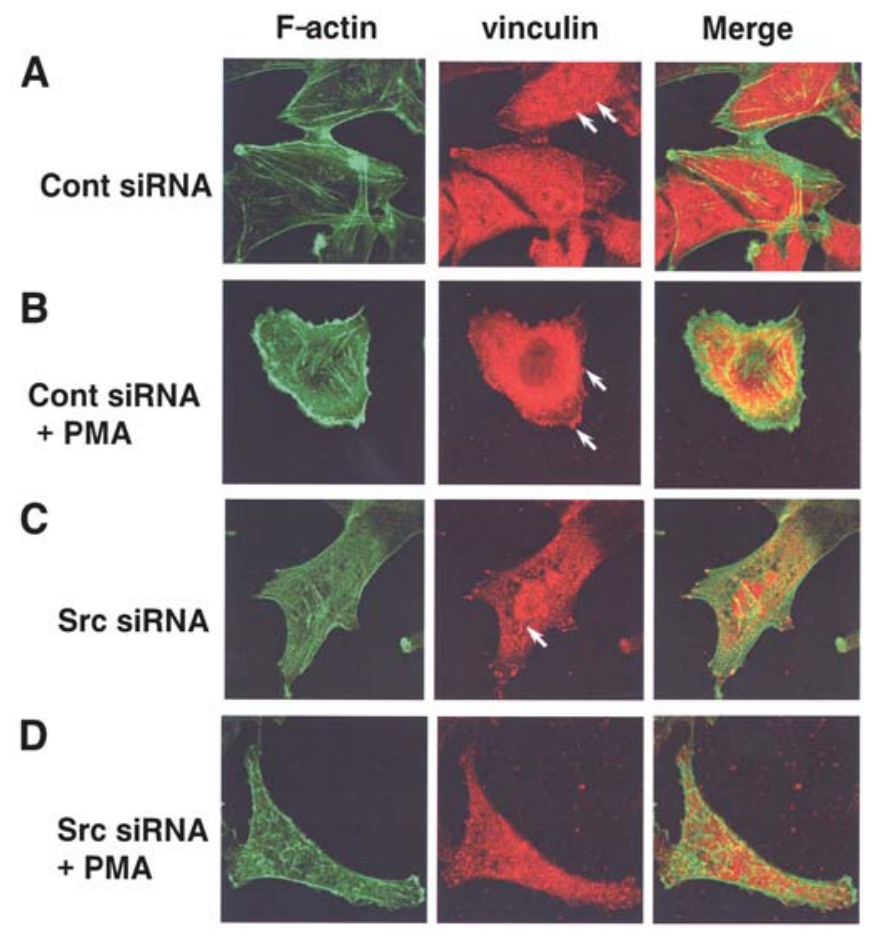

Figure 6. Effect of Src on PMA-induced formation of lamellipodia and focal complexes in glioblastoma cells. Cells were transfected with control (A and B) or Src siRNA (C and D). After $24 \mathrm{~h}$ of serum-starvation, the transfected cells were treated with vehicle (A and C) or PMA (B and D) for $60 \mathrm{~min}$. After fixation, the cells were double-labeled for F-actin and vinculin. F-actin and vinculin were labeled green and red, respectively. Yellow fluorescence shows the region of colocalization of F-actin and vinculin. Arrows indicate the focal complexes.

tyrosine phosphorylated $30 \mathrm{~min}$ after PMA treatment (lane 2), and this phosphorylation was inhibited by PP2 (lane 3).

Furthermore, to confirm the specific effect of Src on the tyrosine phosphorylation of Cas, the cells transfected with Src siRNA were subjected to Western blot analysis. As shown in Fig. 3C (lane 4), tyrosine phosphorylation of Cas was blocked by Src siRNA.

Next we examined the effects of PMA on the molecular association between Src and Cas. Lysates were immunoprecipitated with anti-Src antibody and immunoblotted for Cas and Src to examine the association of Src and Cas. As shown in Fig. 3D, PMA treatment induced formation of Src/ Cas complexes (lane 2) and this phenomenon was inhibited by PP2 (lane 3 ). These results suggested that Src was involved in the PMA-induced tyrosine phosphorylation of Cas.

PMA-induced formation of Cas/Crk complex and subsequent activation of Racl was mediated via Src. Tyrosine phosphorylation of Cas in its substrate-binding YxxP domain results in the generation of multiple binding sites for SH2-containing adapter protein Crk $(8,9,16)$. Cas/Crk complex signaling reactions eventually result in the activation of $\operatorname{Rac} 1(9,17,18)$. On the basis of these findings from previous studies, we next investigated whether PMA stimulation could promote Cas/ Crk complex formation, and if so, whether this Cas/Crk complex formation depends on Src. As shown Fig. 4, immunoprecipitation of Cas from both control (lane 1) and PMAtreated (lane 2) cells revealed that PMA increased the association between Cas and Crk, and this phenomenon was

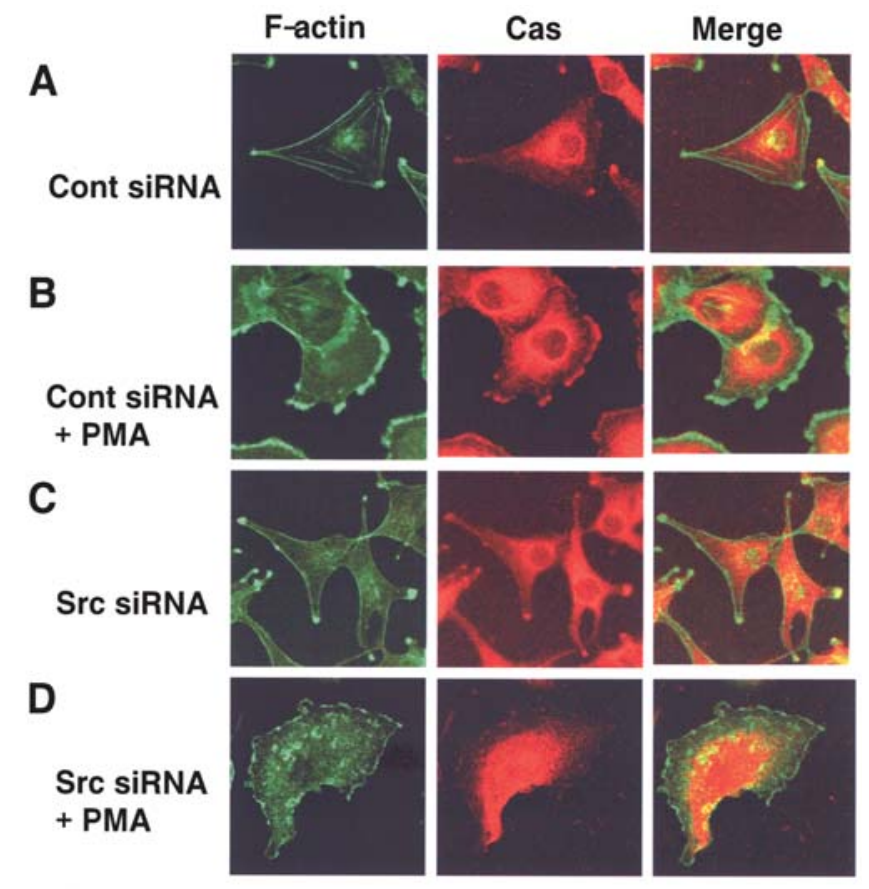

Figure 7. Effect of Src on PMA-induced F-actin formation and Cas localization in glioblastoma cells. Cells were transfected with control (A and B) or Src siRNA (C and D). After $24 \mathrm{~h}$ of serum-starvation, the transfected cells were treated with vehicle (A and C) or PMA (B and D) for $60 \mathrm{~min}$. After fixation, the cells were double-labeled for F-actin and Cas. F-actin and Cas were labeled green and red, respectively. Yellow fluorescence shows the region of colocalization of F-actin and Cas.

abolished by PP2 (lane 3) or BIS (lane 4). These data indicated that $\mathrm{Src}$ activation was involved in PMA-induced Cas/Crk complex formation. Our results were consistent with previous studies that Src was implicated in the PKC-dependenttyrosine phosphorylation of Cas, and following Cas/Crk complex in neuroblastoma cells (19).

To identify the signaling pathway located downstream of Cas/Crk, we next explored whether PMA could activate Rac1, which is known to be one of the downstream effector of Cas/ Crk complex. To detect Rac1 activation, a PAK PBD GST pull-down assay was performed to detect GTP-bound Rac1. As shown in Fig. 5A, Rac1 was activated 5 min after PMA stimulation. Then, to analyze whether Rac1 activation was dependent on Src, the Rac1 activation status was analyzed in the cells treated with PP2 or Src siRNA before PMA treatment. Fig. 5B shows that PMA induced Rac1 activation and this activation was diminished by PP2. Furthermore, in the cells transfected with Src siRNA, PMA-induced Rac1 activation was blocked (Fig. 5C). These data indicated that Src was involved in PMA-induced Rac1 activation in glioblastoma cells.

PMA-induced formation of lamellipodia and focal complexes in the glioblastoma cells was dependent on Src. Previously, we found that PMA-activated PKC induced lamellipodia formation at the leading edge followed by focal complex formation under the lamellipodia in glioblastoma cells (unpublished data). In addition, activation of Rac1 signaling is known to regulate the lamellipodia and focal complex formation $(9,10,20)$. 


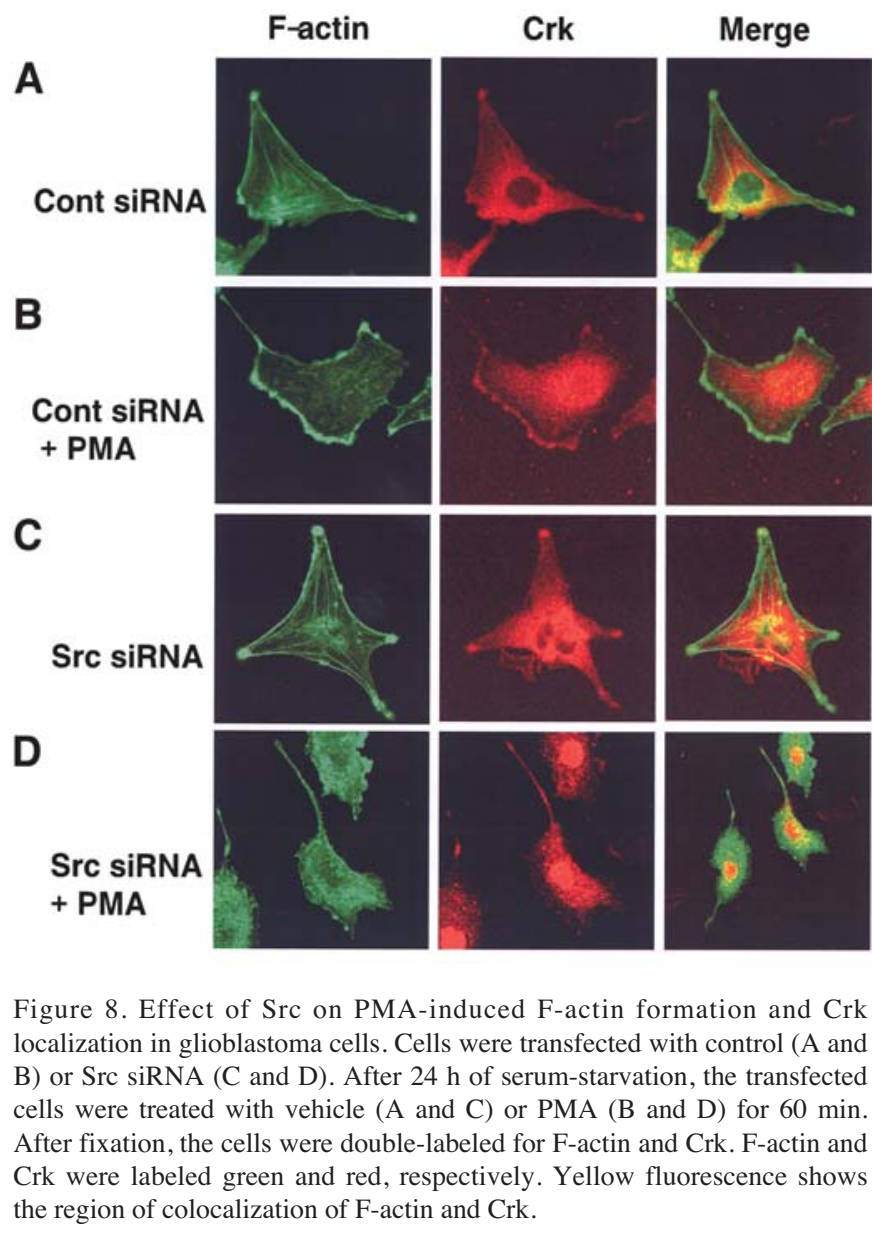

To examine the involvement of Src/Rac1 signaling in PMA-induced formation of lamellipodia and focal complexes, immunohistochemical analysis was performed. To visualize focal adhesions, the cells were stained with vinculin, and were also stained with phalloidin to detect F-actin. In control cells, vinculin staining revealed large focal adhesions at the ends of thick actin cables, which indicated the establishment of stable, cytoskeleton-associated adhesions (Fig. 6A). After PMA treatment, lamellipodia were observed in the cell periphery and vinculin staining revealed that dot-shaped focal complexes were formed under the protrusive lamellipodia in the cells (Fig. 6B).

Then to analyze the involvement of Src in PMA-induced cytoskeletal changes, cells with PP2 pretreatment (data not shown) or Src siRNA transfection (Fig. 6C and D) were analyzed by immunohistochemistry. In both preparations of cells, PMA-induced lamellipodia formation was largely abolished, and vinculin staining showed that large focal adhesions were present in the cytoplasm of PMA-treated cells, as in control cells (Fig. 6D).

PMA-induced translocation of Cas and Crk to cell periphery was dependent on Src. To confirm that Cas and Crk are involved in PMA-induced cell migration, we performed fluorescence immunohistochemistry to analyze the subcellular localization of Cas and Crk. In the control cells, diffusely distributed Cas and Crk were observed in the cytoplasm

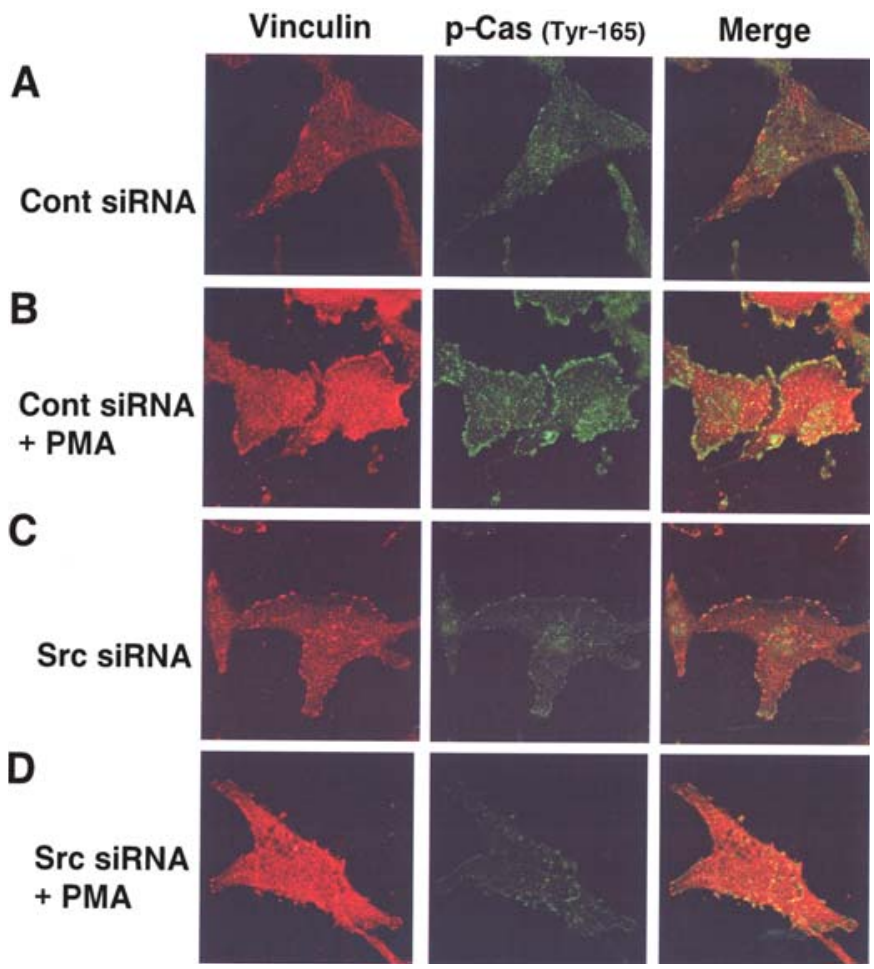

Figure 9. Effect of Src on PMA-induced focal complex formation and phosphorylation of Cas in glioblastoma cells. Cells were transfected with control (A and B) or Src siRNA (C and D). After $24 \mathrm{~h}$ of serum-starvation, the transfected cells were treated with vehicle (A and C) or PMA (B and D) for $60 \mathrm{~min}$. After fixation, the cells were double-labeled for vinculin and phosphorylated-Cas (Tyr-165). Vinculin and phosphorylated-Cas (Tyr-165) were labeled red and green, respectively. Yellow fluorescence shows the region of colocalization of vinculin and phosphorylated Cas (Tyr-165).

(Figs. 7A and 8A). In contrast, Cas and Crk were detected in the lamellipodia upon PMA stimulation (Figs. 7B and 8B). The translocation of Cas or Crk was blocked by BIS (data not shown), indicating that the translocation was dependent on PKC.

Next, we analyzed the role of Src in PMA-induced translocation of Cas and Crk using PP2 or Src siRNA. PMAinduced translocation of Cas and Crk to the lamellipodia was blocked by PP2 (data not shown) or Src siRNA (Figs. 7D and 8D).

Furthermore, to analyze the subcellular localization of tyrosine-phosphorylated Cas, the cells were double-stained with anti-phospho-Cas (Tyr-165) antibody and vinculin to visualize focal complexes. As shown in Fig. 9A, tyrosine phosphorylated Cas was rarely stained in the large focal adhesions in quiescent cells. However, after PMA treatment, tyrosine-phosphorylated Cas was reorganized into focal adhesions in the cytoplasm and also dot-shaped focal complexes at the cell periphery (Fig. 9B). This phenomenon was attenuated by BIS (data not shown) or Src siRNA (Fig. 9D). These results showed that Src was involved in PMA-induced cytoskeletal reorganization through Cas and Crk.

PMA-induced translocation of Racl to the cell membrane was dependent on Src. Previous studies have shown that in addition to GTP loading of Rac1, appropriate subcellular localization of Rac1 is necessary for proper activation of the downstream 
A
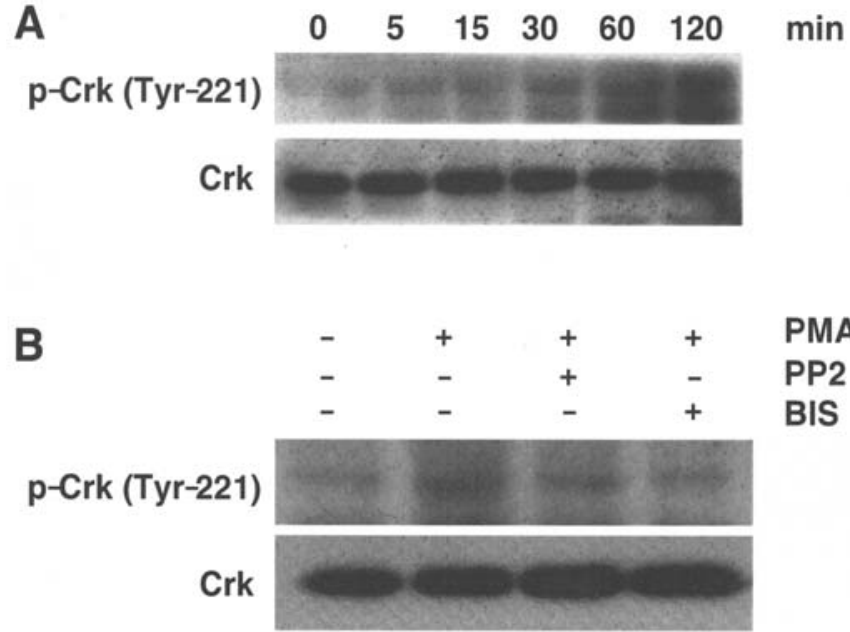

C

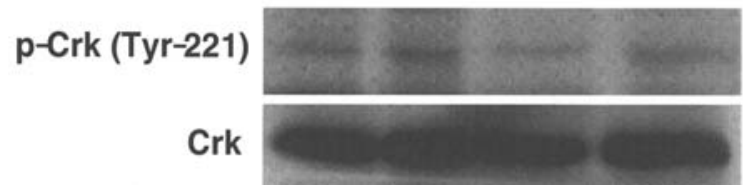

Figure 10. Effect of Src on expression and Tyr-221 phosphorylation of Crk in glioblastoma cells. (A) Cells were treated with PMA for the indicated times. (B) For inhibition experiments, PP2 or BIS was added to serumstarved cells 30 min prior to PMA stimulation. Then the cells were stimulated with PMA or vehicle for $60 \mathrm{~min}$. (C) Cells transfected with control or Src siRNA were incubated without serum for $24 \mathrm{~h}$. Then the cells were stimulated with PMA or vehicle for $60 \mathrm{~min}$. Expression and tyrosine phosphorylation of Crk were examined by Western blot analysis. Phosphorylation of Crk (Tyr-221) was evaluated with an anti-phospho-Crk (Tyr-221) antibody. The amount of total Crk was determined by reprobing the membrane after stripping.

pathway (21-23). It was reported that Rac1 translocation was regulated by the phosphorylation of the Tyr-221 residue of Crk (21). Therefore, we examined the PMA-induced phosphorylation of Crk in glioblastoma cells using a specific antibody that detects Crk only when Tyr-221 is phosphorylated. As shown in Fig. 10A and B, PMA stimulation induced phosphorylation of Crk (Tyr-221) and this phosphorylation was blocked by BIS. To analyze the involvement of Src in the phosphorylation of Crk (Tyr-221), the phosphorylation of Crk (Tyr-221) was examined in the cells treated with PP2 (Fig. 10B, lane 3) or Src siRNA (Fig. 10C, lane 4). PP2 or Src siRNA also blocked the PMA-induced phosphorylation of Crk (Tyr-221).

Then, we analyzed the PMA-induced changes of the subcellular localization of Rac1 by immunohistochemistry. As shown in Fig. 11A, Rac1 was diffusely distributed in the cytoplasm in the control cells. After PMA treatment, Rac1 was observed to translocate into the lamellipodia (Fig. 11B). The translocation of Rac1 was abolished by BIS (data not shown). To analyze the role of Src in Rac1 translocation in the cells, the localization of Rac1 was analyzed in the cells treated with PP2 (data not shown) or transfected with Src

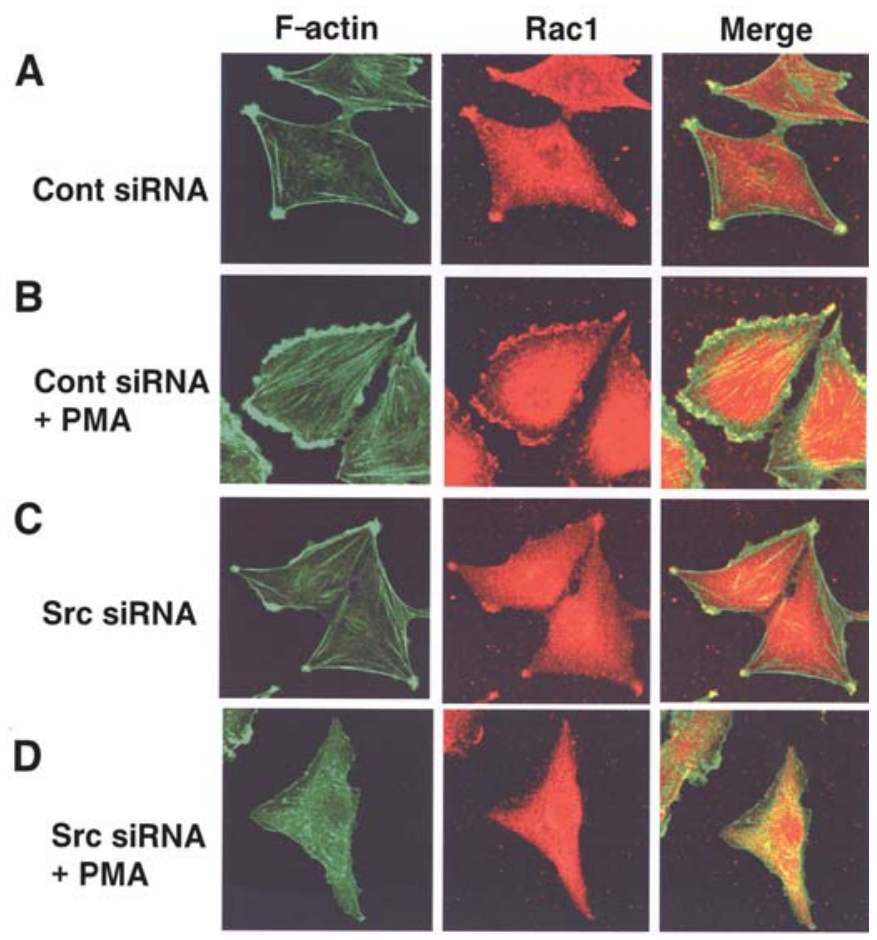

Figure 11. Effect of Src on F-actin formation and Rac1 expression in glioblastoma cells. Cells were transfected with control (A and B) or Src siRNA (C and D). After serum-starvation, the transfected cells were treated with vehicle (A and C) or PMA (B and D) for 60 min. After fixation, the cells were double-labeled for F-actin and Rac1. F-actin and Rac1 were labeled green and red, respectively. Yellow fluorescence shows the region of colocalization of F-actin and Rac1.

siRNA (Fig. 11C and D). As expected, Src siRNA abolished the PMA-induced translocation of Rac1 to the cell periphery. These findings indicated that Src might be involved in the PMA-induced translocation of Rac1 to the cell membrane via phosphorylation of Crk (Tyr-221).

\section{Discussion}

Src plays critical roles in a variety of cellular signal transduction pathways, and regulates diverse processes such as cell division, motility, adhesion, angiogenesis, and survival (24). 12-O-tetradecanoylphorbol-acetate (TPA), a PKC stimulator has been described to result in an increase of Src kinase activity in vivo $(13,25,26)$. Previous observations showed that Src mediates growth factor-induced tyrosine phosphorylation of Cas $(19,27,28)$.

In this study, we examined the role of Src in PMA-induced migration of glioblastoma cells. We proposed that PMAactivated PKC-induced migration was mediated via the Cas/ $\mathrm{Crk} / \mathrm{Rac} 1$ pathway in a manner dependent on Src in glioblastoma cells.

PMA stimulation induced rapid tyrosine phosphorylation of Cas via Src (Fig. 3). Once tyrosine is phosphorylated, Cas exerts its downstream signaling primarily through association with Crk. A previous study showed that TPA-induced phosphorylation of Cas by Src promotes establishment of Cas/Crk complexes dependent on the activity of novel PKCs (19). These data are consistent with our results that PMA-induced Cas/Crk complex formation was dependent on Src (Fig. 4). 
To gain further insight into the events following the activation of Src in migrating cells, we performed immunohistochemistry. Cas and Crk were accumulated in the lamellipodia (Figs. 7B and 8B), and tyrosine-phosphorylated Cas was localized at the focal complexes in the PMA-treated cells (Fig. 9B). These results suggested that signaling of Cas and Crk might be involved in PMA-dependent cytoskeletal reorganization. Furthermore, Src inhibition abolished these phenomena (Figs. 7D, 8D and 9D), indicating that Src might modulate cytoskeletal changes through affecting not only the tyrosine phosphorylation of Cas but also the subcellular localization of Cas and its binding partner, Crk.

A Cas/Crk complex is known to mediate lamellipodia formation and cell migration in a Rac1-dependent manner $(9,17)$. Recent studies demonstrated that PMA increased GTP-loading of Rac1 $(29,30)$. We showed that PMA induced Rac1 activation and Src might regulate PMA-induced activation of Rac1 in glioblastoma cells (Fig. 5B and C).

Activation of the Rac1 signaling pathway is required for both GTP-loading of Rac1 and membrane translocation of Rac1 (21). We confirmed that Rac1 was found in lamellipodia in PMA-induced migratory cells (Fig. 11B). It was reported that the Y221 site in Crk regulates adhesion-dependent membrane localization of both Crk and Rac1, presumably via tyrosine phosphorylation $(21,23)$. Growth factors such as epidermal growth factor (22), nerve growth factor (30), and insulin-like growth factor-1 (31) induce tyrosine phosphorylation of Crk. The mechanism of regulation of phosphorylation of Crk (Tyr-221) is not completely understood, although it has been shown that Crk was phosphorylated in $\mathrm{v}$-Srctransformed 3Y1 (22) or NIH3T3 cells (32). We demonstrated that Crk (Tyr-221) was phosphorylated by PMA treatment, and this phosphorylation was blocked by Src inhibition (Fig. 10B and C). Furthermore, PMA-induced translocation of Rac1 to the cell membrane was also blocked by Src siRNA (Fig. 11D). As mentioned above, our findings indicated that Src might play a role in PMA-induced translocation of Rac1 to the cell membrane through phosphorylation of Crk (Tyr-221).

Regarding the phosphorylation of Crk (Tyr-221), there are two contradictory theories. It was reported that prevention of the phosphorylation of Crk (Tyr-221) inhibited translocation of Rac1 to the cell periphery and resulted in decreased migration $(21,23)$. In contrast, it was reported that prevention of the phosphorylation of Crk (Tyr-221) increased Cas/Crk complex formation and migration $(9,17,18)$. In our study, Cas/Crk complex formation was detected after PMA treatment (Fig. 4), and phosphorylation of Crk (Tyr-221) was also increased by PMA (Fig. 10A). Our results showed that phosphorylation of Crk at (Tyr-221) was detected upon PMA stimulation, coincident with Rac1 translocation to the cell membrane. As previously reported (33), one possible explanation is that phosphorylation of Crk (Tyr-221) might initially induce active transmission signaling of Crk and subsequently induce the dissociation of the Cas/Crk complex by downregulation signals.

We have to note that in addition to the well-characterized interaction between PMA, and classical and novel protein kinase $\mathrm{C}$ isoforms, PMA promotes binding to other receptors lacking kinase activity, including chimaerins. Chimaerins accelerate in vitro the hydrolysis of GTP of Rac and downregulate Rac function. Although the Rac-GAP activity of chimaerins in vitro has been well documented, the cellular effects of these phorbol ester receptors as a regulator of Racmediated functions in cells remains to be almost unexplored (34). Further analysis is needed to clarify whether downregulation of Rac1-GTP levels by Src inhibition was implicated in an effect of chimaerins.

The important question remaining is whether Rac 1 is actually involved in the PMA-induced migration of the glioblastoma cells, because it was reported that TPA failed to activate Rac1 in rat vascular smooth muscle cells (35). We confirmed that PMA-induced migration was inhibited by Rac1 siRNA in A172 glioblastoma cells (unpublished data). These disparate observations suggested that there might be both Rac1 activation-dependent and -independent mechanisms of cell migration in different cell types.

We previously found that PMA mediated lamellipodia formation and cell migration in A172 glioblastoma cells via the PKC/p38MAPK/Hsp27 pathway (36). In the present study, we showed that PMA-induced lamellipodia formation and migration were dependent on the $\mathrm{PKC} / \mathrm{Src}$ pathway. In several studies, Src was shown to be a positive regulator of p38MAPK $(37,38)$. In addition, Rac1 was also reported to activate p38MAPK $(39,40)$. Considering our findings together with the previous studies, there is a possibility that Src or Rac1 activation induced by PMA could cross-talk with the PKC/p38MAPK/Hsp27 pathway.

In summary, we identified a novel molecular mechanism by which Src is involved in PMA-induced human glioblastoma cell migration through activation of the Cas/Crk/Rac1 signaling pathway. Our studies provide a basis for further characterization of the molecular cascades involved in PMAactivated PKC-stimulated glioblastoma migration, which may yield insights useful for the development of new diagnostic and therapeutic applications for this invasive tumor.

\section{Acknowledgements}

This study was supported in part by grants-in-aid for Encouragement of Scientists (Grant No. 18924037 to M.N.) from Japan Society for the Promotion of Science.

\section{References}

1. Berens ME and Giese A: "...those left behind." Biology and oncology of invasive glioma cells. Neoplasia 1: 208-219, 1999.

2. da Rocha AB, Mans DR, Regner A and Schwartsman G: Targeting protein kinase $\mathrm{C}$ : new therapeutic opportunities against high-grade malignant gliomas? Oncologist 7: 17-33, 2002.

3. Bredel $\mathrm{M}$ and Pollack IF: The role of protein kinase $\mathrm{C}(\mathrm{PKC})$ in the evolution and proliferation of malignant gliomas, and the application of PKC inhibition as a novel approach to antiglioma therapy. Acta Neurochir 139: 1000-1013, 1997.

4. Sharif TR and Sharif M: Overexpression of protein kinase C epsilon in astroglial brain tumor derived cell lines and primary tumor samples. Int J Oncol 5: 237-243, 1999.

5. Cary LA, Klinghoffer RA, Sachsenmaier C and Cooper JA: SRC catalytic but not scaffolding function is needed for integrin-regulated tyrosine phosphorylation, cell migration, and cell spreading. Mol Cell Biol 22: 2427-2440, 2002.

6. Fincham VJ and Frame MC: The catalytic activity of Src is dispensable for translocation to focal adhesions but controls the turnover of these structures during cell motility. EMBO J 17: $81-92,1998$. 
7. Matsuda M, Mayer BJ, Fukui Y and Hanafusa H: Binding of transforming protein, $\mathrm{P} 47 \mathrm{gag}$-crk, to a broad range of phosphotyrosine-containing proteins. Science 248: 1537-1539, 1990.

8. Bouton AH, Riggins RB and Bruce-Staskal PJ: Functions of the adapter protein Cas: signal convergence and the determination of cellular responses. Oncogene 20: 6448-6458, 2001.

9. Klemke RL, Leng J, Molander R, Brooks PC, Vuori K and Cheresh DA: CAS/Crk coupling serves as a 'molecular switch' for induction of cell migration. J Cell Biol 140: 961-972, 1998.

10. Burridge $\mathrm{K}$ and Wennerberg $\mathrm{K}$ : Rho and Rac take center stage. Cell 116: 167-179, 2004

11. Kraynov VS, Chamberlain C, Bokoch GM, Schwartz MA, Slabaugh S and Hahn KM: Localized Rac activation dynamics visualized in living cells. Science 290: 333-337, 2000.

12. Nomura M, Nomura N and Yamashita J: Geldanamycin-induced degradation of Chk1 is mediated by proteasome. Biochem Biophys Res Commun 335: 900-905, 2005.

13. Brandt DT, Goerke A, Heuer M, et al: Protein kinase C delta induces Src kinase activity via activation of the protein tyrosine phosphatase PTP alpha. J Biol Chem 278: 34073-34078, 2003.

14. Fincham VJ, Brunton VG and Frame MC: The SH3 domain directs acto-myosin-dependent targeting of $\mathrm{v}$-Src to focal adhesions via phosphatidylinositol 3-kinase. Mol Cell Biol 20: 6518-6536, 2000

15. Hamasaki K, Mimura T, Morino N, et al: Src kinase plays an essential role in integrin-mediated tyrosine phosphorylation of Crk-associated substrate p130Cas. Biochem Biophys Res Commun 222: 338-343, 1996.

16. Sakai R, Iwamatsu A, Hirano N, et al: A novel signaling molecule, p130, forms stable complexes in vivo with v-Crk and $\mathrm{v}-\mathrm{Src}$ in a tyrosine phosphorylation-dependent manner. EMBO J 13: 3748-3756, 1994.

17. Cherersh DA, Leng J and Klemke RL: Regulation of cell contraction and membrane ruffling by distinct signals in migratory cells. J Cell Biol 146: 1107-1116, 1999.

18. Cho SY and Klemke RL: Purification of pseudopodia from polarized cells reveals redistribution and activation of Rac through assembly of a CAS/Crk scaffold. J Cell Biol 156: 725-736, 2002.

19. Bruce-Staskal and Bouton AH: PKC-dependent activation of FAK and src induces tyrosine phosphorylation of Cas and formation of Cas-Crk complexes. Exp Cell Res 264: 296-306, 2001.

20. Rottner K, Hall A and Small JV: Interplay between Rac and Rho in the control of substrate contact dynamics. Curr Biol 9: 640-648, 1999

21. Abassi YA and Vuori K: Tyrosine 221 in Crk regulates adhesiondependent membrane localization of Crk and Rac and activation of Rac signaling. EMBO J 21: 4571-4582, 2002.

22. Hashimoto Y, Katayama H, Kiyokawa E, et al: Phosphorylation of CrkII adaptor protein at tyrosine 221 by epidermal growth factor receptor. J Biol Chem 273: 17186-17191, 1998.

23. del Pozo MA, Vicente-Manazanares M, Tejedor R, Serrador JM and Sanchez-Madrid F: Rho GTPases control migration and polarization of adhesion molecules and cytoskeletal ERM components in T lymphocytes. Eur J Immunol 29: 3609-3620, 1999.

24. Playford MP and Schaller MD: The interplay between Src and integrins in normal and tumor biology. Oncogene 23: 7928-7946, 2004.
25. Schlaepfer DD, Jones KC and Hunter T: Multiple Grb2-mediated integrin-stimulated signaling pathways to ERK2/mitogenactivated protein kinase: summation of both $\mathrm{c}-\mathrm{Src}$ - and focal adhesion kinase-initiated tyrosine phosphorylation events. Mol Cell Biol 18: 2571-2585, 1998.

26. Xian W, Rosenberg MP and Di Giovanni J: Activation of erbB2 and c-src in phorbol ester-treated mouse epidermis: possible role in mouse skin tumor promotion. Oncogene 14: 1435-1444, 1997.

27. Nakamoto T, Sakai R, Ozawa K, Yazaki Y and Hirai H: Direct binding of C-terminal region of p130Cas to $\mathrm{SH} 2$ and $\mathrm{SH} 3$ domains of Src kinase. J Biol Chem 271: 8959-8965, 1996.

28. Klinghoffer RA, Sachsenmier C, Cooper JA and Soriano P: Src family kinases are required for integrin but not PDGFR signal transduction. EMBO J 18: 2459-2471, 1999.

29. Caloca MJ, Wang H and Kazanietz MG: Characterization of the Rac-GAP (Rac-GTPase-activating protein) activity of beta2chimaerin, a 'non-protein kinase C' phorbol ester receptor. Biochem J 375: 313-321, 2003.

30. You HJ, Woo CH, Choi EY, Cho SH, Yoo YJ and Kim JH: Roles of Rac and p38 kinase in the activation of cytosolic phospholipase A2 in response to PMA. Biochem J 388: 527-535, 2005

31. Zhu T, Goh EL, LeRoith D and Lobie PE: Growth hormone stimulates the formation of a multiprotein signaling complex involving p130(Cas) and CrkII. Resultant activation of c-Jun $\mathrm{N}$-terminal kinase/stress-activated protein kinase (JNK/SAPK). J Biol Chem 273: 33864-33875, 1998.

32. Cheng CH, Yu KC, Chen HL, et al: Blockade of v-Srcstimulated tumor formation by the Src homology 3 domain of Crk-associated substrate (Cas). FEBS Lett 557: 221-227, 2004.

33. Chodniewicz D and Klemke RL: Regulation of integrinmediated cellular responses through assembly of a CAS/Crk scaffold. Biochim Biophys Acta 1692: 63-76, 2004.

34. Ron D and Kazanietz MG: New insights into the regulation of protein kinase $\mathrm{C}$ and novel phorbol ester receptors. FASEB J 13: $1658-1676,1999$.

35. Brandt D, Gimona M, Hillmann M, Haller $\mathrm{H}$ and Mischak H: Protein kinase $\mathrm{C}$ induces actin reorganization via a Src- and Rho-dependent pathway. J Biol Chem 277: 20903-20910, 2002.

36. Nomura N, Nomura M, Sugiyama K and Hamada J: Phorbol 12-myristate 13-acetate (PMA)-induced migration of glioblastoma cells is mediated via p38MAPK/Hsp27 pathway. Biochem Pharmacol (In press).

37. McMulluen M, Keller R, Sussman M and Pumiqlia K: Vascular endothelial growth factor-mediated activation of p38 is dependent upon Src and RAFTK/Pyk2. Oncogene 23: 12751282,2004

38. Nagao M, Yamauchi J, Kaziro Y and Itoh H: Involvement of protein kinase $\mathrm{C}$ and Src family tyrosine kinase in Galphaq/11induced activation of $\mathrm{c}-\mathrm{Jun} \mathrm{N}$-terminal kinase and $\mathrm{p} 38$ mitogen-activated protein kinase. J Biol Chem 273: 2289222898, 1998.

39. Buchsbaum RJ, Connolly BA and Feig LA: Interaction of Rac exchange factors Tiam1 and Ras-GRF1 with a scaffold for the p38 mitogen-activated protein kinase cascade. Mol Cell Biol 22: 4073-4085, 2002

40. Baldassa S, Gnesutta N, Fascio U, Sturani E and Zippel R: SCLIP, a microtubule-destabilizing factor, interacts with RasGRF1 and inhibits its ability to promote Rac activation and neurite outgrowth. J Biol Chem 282: 2333-2345, 2007. 